\title{
Development and application of artificial intelligence for military training modeling and simulation in Republic of Korea
}

\author{
Youngmook $\mathrm{Kim}^{*}$
}

\begin{abstract}
In the era of the Fourth Industrial Revolution, efforts are being made throughout society to achieve innovation by utilizing artificial intelligence (Al). Developed countries are trying to maximize their combat capabilities using $\mathrm{Al}$ in defense areas.

Al research on the defense of the Republic of Korea is focused on field-related applications, such as drones and robots; however, the research on the application of Al to training fields is relatively insufficient. In this paper, this study briefly summarizes the concept of $\mathrm{Al}$ and examines the use of Al in modeling and simulation (M\&S) training, one of the defense M\&S fields. Furthermore, the present study evaluates the areas where development is possible using current technology: decision support systems, intelligent tutoring systems, and automatic simulation of virtual entities.
\end{abstract}

Keywords : artificial intelligence, training modeling and simulation, decision-making support system, intelligent tutoring system, automated simulation of virtual entities

(1) (1) This work is licensed under a Creative Commons Attribution 4.0 International License.

* (First Author) ROK (Republic of Korea) Combined Forces Command, Major (Chungnam National University, department of business administration, Ph.D. candidate), dudanr92@gmail.com 


\section{I. 서론}

4차 산업혁명이라는 키워드가 국방, 산업, 의료 등 다양한 분야에서 화두가 되고 있다. 특히 4차 산업혁명의 핵심 기술 중 하나인 인공지능은 미래 우리의 삶을 크게 바꿀 것으로 기대되고 있다. 지금의 인공지능은 기계학습(machine learning)과 딥러닝(deep learning)의 발전으로 시작되었고, $\mathrm{IBM}$ 의 왓슨 프로젝트,1) 알파고와 이세돌의 바둑 대결, 레이 커즈와일 같은 미래학자들의 특이점 (singularity)2)에 대한 주장들에 의해 더욱 큰 관심을 받고 있다. 한편, 국방 분야는 드론봇 등에 관 한 연구가 활발하게 진행되고(Eom, 2021; Yoon \& Choi, 2020), 여러 가지 시범사업 등을 추진하고 있다.3) 하지만, 교육훈련 분야에서 관련 연구가 상대적으로 부족하여 향후 첨단 인공지능 기술을 활용한 교육훈련 분야의 연구가 요구된다.

이런 필요성에 따라, 본 연구는 인공지능에 대한 기본적인 개념 및 특징을 제시하고, 교육훈련 $\mathrm{M} \& S$ 분야4)에 인공지능을 구현하기 위한 기법 적용 사례를 탐색적으로 고찰해 본다. 이를 바탕으 로 미래에 인공지능 기법을 활용하여 교육훈련 성과 극대화 방안을 제시함으로써 군의 훈련 효과 측면에서 학문적 발전에 필요한 기초자료를 제공하고자 한다.

\section{ㅍ. 인공지능의 개념}

1950년 앨런 튜링(Alan Mathison Turing)에 의해 인공지능의 개념이 처음 제안된 후, 1956년 미 국 다트머스에서 개최한 세미나에서 존 매카시에 의해 '인공지능(artificial intelligence)'이라는 단 어가 처음으로 사용되었다. 그 후, 인공지능에 대한 정의는 시대와 학자에 따라 다양하게 정의하고 있으나, 러셀과 노빅이 정의한 '인간처럼 행동하는 시스템'이 직관적으로 이해가 쉽다. 이 정의에 따르면 인공지능은 인간처럼 몸과 마음이 필요한데, 몸에 해당하는 부분이 컴퓨터 또는 하드웨어이 고 마음에 해당하는 부분이 알고리즘 또는 소프트웨어이다. 따라서 인공지능의 발전은 필연적으로 컴퓨터와 알고리즘의 발전사와 밀접하게 관련이 있으며, 인공지능과 인공지능 기법 등은 인간처럼 행동하기 위해 만들어진 내용을 포함하고 있다.

다음으로 인공지능과 기계학습, 딥러닝의 개념적 관계를 구분하면, 기계학습은 인공지능을 구현

1) 자연어 형식으로 된 질문들에 답할 수 있는 인공지능 컴퓨터 시스템.

2) 인공지능이 비약적으로 발전해 인간의 지능을 뛰어넘은 시점으로 레이 커즈와일은 2045년쯤에 될 것으로 예측함.

3) 육군 교육사령부(2020), 20-1차 인공지능 추진사업 공개설명회.

4) M\&S는 Modeling and Simulation의 약자로서 Modeling은 현실 세계를 의도된 목적에 따라 단순화/이상화/추상화하여 표현하는 작업이며, Simulation은 그 표현한 것을 시간의 흐름에 따라 순차적으로 구현하는 방법임. 국방 M\&S 활용 분야는 훈련, 분석, 획득의 3 가지로 분류됨. 
Development and application of artificial intelligence (Al) for military training modeling and ... / Youngmook Kim 23

하는 방법인 전문가 시스템 등과 같은 하나의 방법론이다. 반면, 딥러닝은 기계학습을 구현하기 위 한 여러 가지 방법의 하나인 인공신경망 기법이 발전한 것으로 구분할 수 있다(Figure 1).

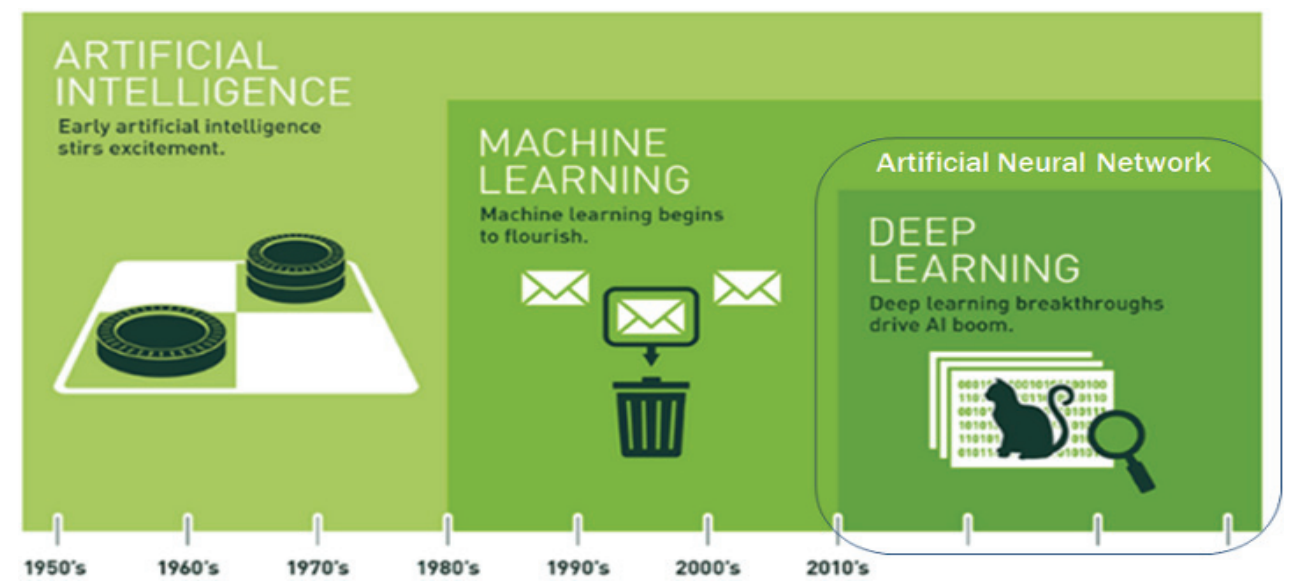

$<$ Figure $1>$ Concept maps of artificial intelligence, machine learning, and deep learning

* source: NVIDIA website from https://developer.nvidia.com/deep-learning

전문가 시스템은 과거에 인공지능의 전성기를 주도했던 기법으로 어떤 전문 분야에 대한 지식을 받아들여 추론하는 프로그램이다. 이런 시스템은 일반적으로 전문가의 지식과 경험을 사실과 규칙 의 형태로 저장한 지식 데이터베이스, 자문과 의사결정을 생성하는 추론 엔진, 사용자와 시스템 간 의 입력/출력 인터페이스로 구성이 된다. 1970년대 초에 스탠퍼드 대학교에서 만든 마이신(Mycin) 이 대표적인 전문가 시스템이다.

Figure 2처럼 전문가에 의해 저장된 지식 데이터베이스가 있고, 전문가와 사용자 사이에 추론 엔

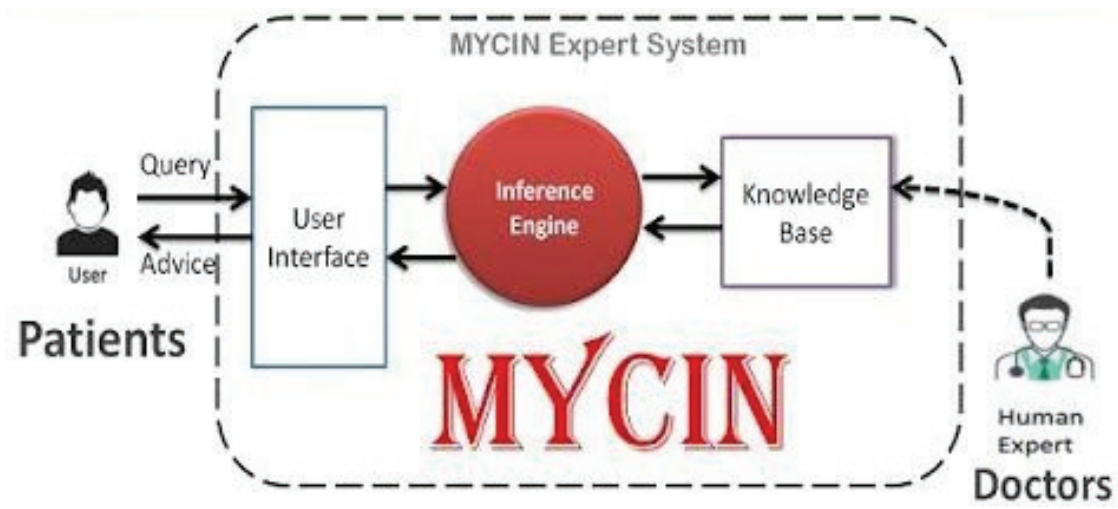

$<$ Figure 2> Expert systems in artificial intelligence with Mysin

* source: https://www.youtube.com/watch?v=ipEOjuV0vns 
진이 존재하며, 사용자와 의사소통하는 인터페이스가 있어 질문하고 답변을 받는다. 마이신은 500 여 개의 규칙이 정해져 있어서 질문에 답을 하면서 따라가다 보면 감염된 세균을 정하고 그에 따른 처방을 할 수 있는 시스템이었다. 하지만 이런 전문가 시스템은 새로운 지식의 업데이트 문제, 개인 용 컴퓨터의 보급 등의 이유로 성장 동력이 줄어들었고, 점차 그 매력이 줄어들게 되었다.

\section{1 기계학습(machine learning)}

기계학습은 IBM의 연구원이었던 아서 사무엘(Arthur Lee Samuel)이 1959년 자신의 논문에서 처음으로 사용한 단어로 컴퓨터에 명시적으로 프로그램을 하지 않고도 컴퓨터가 학습할 수 있는 능력을 제공하는 연구 분야로 정의했다. 쉽게 말해 컴퓨터가 스스로 표본이 되는 데이터를 바탕으 로 규칙이나 지식을 스스로 학습하는 것을 의미한다. 학습 방법에 따라 지도학습, 비지도학습의 2 가지로 분류하거나, 강화학습까지 포함하여 3 가지로 분류한다. 1990년대의 기계학습은 컴퓨터를 이 용한 통계학에 가까웠으며, 통계학 관점에서 데이터를 분석하는 데이터마이닝과 많은 부분을 공유 했다. 최근 가장 주목받는 인공지능 기술 분야인 딥러닝은 기계학습을 구현하기 위한 방법 중 하나 인 인공신경망 기법이 발전한 것이다.

\subsection{1 지도학습(supervised learning)}

지도학습은 입력값과 올바른 출력값을 세트화한 데이터를 미리 준비하고, 어떤 입력이 주어졌을 때 올바른 출력이 나오도록 학습을 하는 것을 말한다. 출력값이 어떤 그룹에 속할 것인지를 판단하 는 것을 분류(classification) 모델이라고 하고, 어떤 출력값이 나올 것인지를 판단하는 것을 예측 (prediction)모델이라고 한다.

분류 모델의 시초라고 볼 수 있는 것이 1936년 영국의 통계학자 피셔가 붓꽃을 4가지 특성으 로 구분하는 방법을 제시한 것이었다. 피셔는 세토사, 버시컬러, 버지니카의 3 가지 종류의 붓꽃 종을 각각 50 개의 표본으로 추출한 후, 꽃받침의 길이와 넓이, 꽃잎의 길이와 넓이를 기록한다. 즉, 이 경우에는 입력값이 붓꽃의 4 가지 특성이 되고, 출력값이 3 가지의 붓꽃종이 된다. 적절한 모델링을 통해서 어떤 붓꽃의 4가지 특성이 입력되면 어떤 종에 해당하는지 분류할 수 있는 것 이다.

최근 분류의 기법이 적용된 예로 이메일의 내용을 확인하여 스팸메일인지 아닌지 여부를 판단하 는 것이 있다. 분류를 위한 대표적인 알고리즘에는 $\mathrm{kNN}(\mathrm{k}$-Nearest Neighbor), $\mathrm{SVM}$ (Support Vector Machine), 의사결정 트리 등이 있다. 예측모델에 관한 예를 들면, 중대원의 키와 몸무게 자 료를 이용하여 키와 몸무게를 잘 설명할 수 있는 함수를 구한 후에, 키가 $175 \mathrm{~cm}$ 인 중대원의 몸무게 를 알아낼 수 있다. 이런 모델의 대표적인 형태가 회귀모델이다. 


\subsection{2 비지도학습(unsupervised learning)}

비지도학습은 지도학습과는 다르게 입력값만 있는 상태에서 학습하는 방법으로 입력값의 구조 적 특성을 파악할 수 있다. 데이터 속에 있는 일정한 패턴이나 룰을 찾는 것을 목적으로 군집 (clustering)이 대표적이다.

비지도학습의 예로는 최근 마케팅 분야에서 시장 및 고객 분류, 유튜브에서 맞춤형 동영상의 추 천, 페이스북과 같은 소셜 네트워크 서비스에서 관심사가 같은 사용자를 위한 커뮤니티 등을 추천 할 때 사용할 수 있다. 대표적인 알고리즘으로는 k-means, k-medoids, DBSCAN 등이 있다(Ansari, Azeem, Ahmed, \& Babu, 2015; Arbin, Suhaimi, Mokhtar, \& Othman, 2015).

\section{1 .3 강화학습(reinforcement learning)}

강화학습은 지도학습에 속한 모델로 분류하기도 하고, 강화학습 자체의 독립적인 영역으로 분 류하기도 한다. 지도학습으로 분류되는 이유는 학습 중에 환경으로부터 보상과 벌칙을 지도받아 학습하기 때문이다. 한편 독립적으로 분류되는 이유는 다른 전형적인 지도학습처럼 사전에 사람 으로부터 가이드를 받고 학습하지 않고, 사람이 아닌 환경으로부터 보상과 벌칙을 받아 학습하기 때문이다.

강화학습은 주어진 환경에서 상태를 관측하고 이에 따른 적절한 행동을 하면 이 행동을 기준으 로 환경으로부터 보상을 받는다. 즉, 일련의 '관측-행동-보상'의 상호작용을 반복하면서 환경으로 부터 받는 보상을 최대화하기 위한 태스크(일) 수행해야 하는데 이 과정이 바로 강화학습인 것이다 (Sutton, 1992).

강화학습이 우리에게 익숙한 이유는 바로 이세돌 9단을 꺾은 알파고의 핵심 알고리즘이 바로 이 강화학습이기 때문이다. 알파고는 인터넷에 공개된 기보를 토대로 기본적인 바둑 정석을 익힌 후 무수히 많은 시행착오를 통해 스스로 바둑을 학습했다. 그 결과 지금은 인간의 수준을 넘어서는 바 둑 실력을 갖추게 되었다. 이런 시행착오를 기반으로 학습하는 과정이 사람의 학습 방법과 매우 닮 아 인공지능을 가장 잘 나타내는 모델이라고 주장하는 사람도 있다. 이런 강화학습 모델은 게임이 나 로보틱스에 가장 효과적으로 활용되고 있다. 대표적인 알고리즘으로는 마코프 보상 프로세스, 마코프 디시즌 프로세스 등이 있다.

\subsection{4 딥러닝(deep learning)}

딥러닝은 뇌를 구성하는 신경세포인 뉴런의 동작 원리에 기초한 인공신경망의 개념에서 발전한 것이다. 인공신경망은 사람의 두뇌와 비슷한 방식으로 정보를 처리하는 알고리즘으로 컴퓨터과학, 의학, 심리학 등의 여러 학문을 융합하여 탄생한 개념이다. 최근 컴퓨터 처리 능력과 엄청난 데이터 양의 확보가 가능해짐에 따라서 큰 관심을 받는 기술이다.

기계학습에서 학습데이터의 특성을 정의해야 했던 방법들과는 달리 딥러닝에서는 이런 것들을 
컴퓨터 스스로 할 수 있다는 것이 가장 큰 장점이다. 인간처럼 컴퓨터가 데이터를 이용하여 스스로 학습할 수 있다는 것이다. 예를 들어, 앞에서 지도학습의 예로 들었던 붓꽃을 분류하는 경우에 사람 이 4 가지 특성값을 입력하고 컴퓨터가 붓꽃을 분류하는 판별식을 만들었다. 하지만 딥러닝 알고리 즘을 이용하면 많은 양의 붓꽃 사진을 입력하고 컴퓨터 스스로 특성을 찾아내고 판별해 내는 것이 가능하다.

기계학습의 핵심은 분석하고자 하는 대상의 특성을 선정하는 것인데, 딥러닝 기법을 통해 결과 예측에 가장 적절한 특성을 찾아낼 수 있다. 딥러닝이 최근 핵심적으로 활용되는 분야는 사진 인식, 자연어 처리, 패턴 인식 등의 분야이며 앞으로 활용 분야가 무궁무진할 것으로 기대된다.

\section{III. 훈련 $\mathrm{M} \& \mathrm{~S}$ 분야 인공지능 기법 적용}

앞서 제시된 인공지능의 개념과 활용을 토대로 현재 훈련 M\&S 분야에 활용하고 있는 사 - 군단 전투지휘훈련 모델인 창조21모델과 미군의 $\mathrm{SAF}$ (Semi Automated Forces)에 적용된 인공지능 기 법 적용의 사례를 분석하였다.

\section{1 창조21모델}

창조21모델은 사 - 군단급 지휘관 및 참모의 상황조치 능력을 배양하고, 제대별 통합전투수행능 력을 향상시키기 위해 사용되는 훈련용 모델이다(Lee, Park, \& Kim, 2019; Han, Moon, Choi, \& $\mathrm{Yu}, 2019)$. 지휘소에서 작전계획에 의해 명령을 하달하면, 청군 및 대항군 게이머는 명령을 입력하 고 컴퓨터 계산에 의해 결과값이 출력된다. 가상의 전투현장인 모의반에서는 현 상황을 지휘소로 보고하고, 지휘관 및 참모는 보고된 현황을 통해 작전수행과정에 의한 통합전투를 수행하게 된다. 이런 절차로 진행되는 전투지휘훈련의 특성상 청군 및 대항군을 운용할 인원이 반드시 필요하다. 즉, 부대를 이동시키거나 교전 또는 보급을 위해서는 사람의 개입이 필요한 것이다.

창조21모델에서는 부대가 도로이동을 할 경우, 사용자에 의해 경로가 입력되면 도로 경로를 고 려하여 최단 경로로 이동하게 된다. 이때 Dijkstra 알고리즘이 이용되는데, 이는 비디오 게임에도 많이 사용되는 가장 기초적인 인공지능 기법 중 하나이다. 또한, 2010년까지 근접전투 피해평가 계 산을 위해 미군에서 만든 COBRA(Combat Outcome Based on Rules for Attrition)라는 근접전투 전문가 시스템을 사용하였다. 이 시스템은 앞에서 살펴본 전문가 시스템에 의해 만들어진 것이었 다. 창조21모델의 핵심기능 중 하나인 근접전투 피해평가는 Table 1 과 같은 수식으로 계산이 이루 어지는데, 이 중 사격가담률 승수와 취약성 승수를 구해주는 것이 COBRA 시스템이다. 
Development and application of artificial intelligence (Al) for military training modeling and ... / Youngmook Kim 27

$<$ Table 1> Calculation equation for battle damage assessment of the 'Chang-Jo21' model

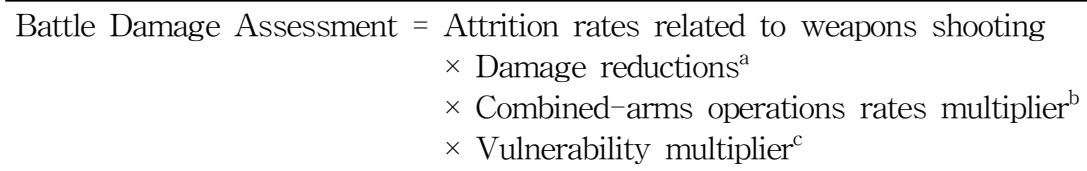

* note. source from Kim, H. S. et al. (2010) and Moon (2018)

a. factors which affect the temporary suppression (e.g., target(s) hid by smoke screen/haze, night action) rather than destruction of weapons

b. abilities regarding combined arms and target acquisition

c. considerations of the concealment of enemy and tactical march intervals

모델에서 부대 간에 교전이 발생하면, 현실성 있는 피해평가의 제공과 훈련 종료 이후에 이 국면 에서의 전투의 결과가 왜 이렇게 나왔는지 분석이 이루어져야 하는데, 이것을 가능하게 한 것이 바 로 COBRA 시스템이었다. COBRA 시스템을 만들기 위해 해당 분야 지식 전문가들에 의해 전술적 고려요소가 피해평가에 미치는 영향을 분석하여 규칙이 만들어졌다. 그 후에 이런 지식들을 컴퓨터 에서 구현 가능한 알고리즘의 형태로 변환하여 모델에서 하나의 모듈로 사용한 것이다. 교전이 시 작되면 COBRA에 사격부대와 피격부대 관련 내용, 지형 관련 사항 등의 63가지 항목이 입력된다. 이 값들을 이용하여 $\mathrm{COBRA}$ 에서는 12 가지 규칙집합에 따라 사격가담률 승수와 취약성 승수 등을 출력하고, 이 값들을 이용하여 피해평가를 실시하게 되며, 미군의 COBRA 시스템을 대체하기 위한 한국형 근접전투 전문가 시스템을 제안하였다(Kim, Moon, Lee, \& Hwang, 2010). 해당 시스템은 다양한 전장 상황이 반영된 640가지 입력값과 출력값의 데이터 세트를 이용하여, 입력값에 대한 출 력값이 기존 근접전투 전문가 시스템과 유사한 결과가 나오도록 하는 의사결정나무를 제안하였다. 여기에 사용된 의사결정나무는 지도학습의 대표적인 알고리즘이다. 현재 창조21모델은 이런 한국 형 근접전투 전문가 시스템을 2011년부터 사용해 오고 있다.

\section{2 $\mathrm{SAF}$ (Semi Automated Forces)}

미군은 1980 년 초까지 단일 무기체계별 시뮬레이터를 활용하여 주로 승무원의 장비조작 훈련 등 을 실시하였으며, 1983년부터 SIMNET(SIMulation NETwork)란 팀 프로젝트로 확장하였으나(Hill \& Miller, 2017; Miller \& Thorpe, 1995) 연결되는 시뮬레이터들이 많아질수록 훈련에 소요되는 비용 은 많이 증가하였다. 특히 복잡하고 큰 부대의 훈련을 실시하는 경우에는 실전적인 상황 조성을 위 해 훈련부대 이외에도 대항군이나 인접부대 및 상급부대 등의 묘사가 필요하였다. 하지만 이런 역 할을 위해 장비와 인원을 쓰는 것은 너무나 큰 예산의 낭비였기 때문에 이런 역할을 할 수 있는 $\mathrm{SAF}$ 가 1988 년까지 개발되었다.

그 이후 1993년에는 재사용성이 용이하고 다양한 인원들이 사용하도록 오픈소스 형태인 Mod-SAF 가 개발되었다. 이를 이용하여 각자의 조직에서 필요로 하는 $\mathrm{SAF}$ 가 개발되었고, 이에 따라 다양한 
형태의 SAF를 하나의 모듈로 사용할 수 있도록 하는 OneSAF가 1996년에 개발되어 활용되었다. OneSAF는 한 명의 조작인원이 많은 수의 객체 생성 및 현실적 조작이 가능하였고, 피훈련자는 컴 퓨터에 의해 움직이는 것인지 사람에 의해 조작이 이루어지는 것인지 거의 인지하지 못하였다. 이 후 다른 나라 및 업체 등은 이런 OneSAF 프로젝트를 그들의 고유한 인공지능 기법(예: JSAF, Abacus, VR Force, Masa Sword, VBS3 등)이 적용된 SAF 또는 워게임 모델로 만들기 시작하 였다.

\section{IV. 인공지능 기법을 활용한 훈련 분야의 발전 방안}

훈련 분야에 인공지능 기법을 사용한 사례는 상기한 바처럼 대부분 워게임 모델에 국한되어 있 으며, 그 외에 교육훈련 분야에 활용한 사례는 거의 찾아보기 힘들다. 하지만 최근 사회 환경의 변 화, 과학화훈련체계의 필요성, 컴퓨터 기술의 발전 등으로 인해 훈련 M\&S 분야에 인공지능 기법 활 용의 필요성이 증대되고 있어 최신의 인공지능 기법을 활용한 교육훈련 분야의 획기적인 발전 가 능성을 높일 수 있다. 이에 본 연구는 훈련 M\&S에서 의사결정 지원체계, 지능형 교관, 가상 개체 자동 모의 분야를 중심으로 인공지능 기법이 적용된 우리 군의 구체적인 발전 방안을 제시하고자 한다.

\section{1 의사결정 지원체계}

의사결정이란 불확실한 환경과 제한된 합리성을 극복하여 원하는 결과에 도달하고자 하는 행위 로, 종종 문제해결의 과정으로 정의된다. 전장에서 지휘자 또는 지휘관은 의사결정의 상황에 수도 없이 직면하며, 올바른 의사결정을 해야 인명 피해를 최소화할 수 있다. 인공지능을 의사결정 지원 체계에 활용한다면 적시적이고 정확한 상황판단과 의사결정에 도움이 될 것이다.

미군의 의사결정 지원체계는 MPARS(Mission Planning and Rehearsal System)이 있다. 이것은 2001년 9.11테러 직후에 00공정사단에 소요를 제기한 것으로, 작전에 투입되기에 앞서 최상의 방책 을 선정하여 작전명령으로 발전시키고 지휘관/참모의 작전절차 숙달을 목적으로 사전 예행연습을 지원할 수 있는 M\&S 기반의 지휘통제 지원체계이다. 주요 역할은 작전명령을 입력받아 예행연습 최초상황을 합성으로 조성하고 자율지능 대항군을 제공하는 것이다. 다음으로 첨단 의사결정 지원 체계인 Deep Green이 있는데, 이것은 2008년 DARPA가 발주한 사업의 명칭이었다. 기존 의사결정 패러다임인 $\mathrm{OODA}$ 5)가 순차적으로 수행되기 때문에 작전의 적시성 보장이 제한되는 문제점을 해 결하기 위해 만들어진 체계가 바로 Deep Green이었다. 이는 전투지휘 의사결정을 지원하며. 지휘

5) Observe-Orient-Decide-Act의 순서로 진행되는 의사결정 체계. 
Development and application of artificial intelligence (Al) for military training modeling and ... / Youngmook Kim 29

관 및 참모의 방책 개발을 보좌하기 위한 의사결정 지원체계이다. 이를 위해 지휘관의 명령을 입력 받는 Commander's Associate, 입력된 명령을 이용하여 교전의 결과를 예측해주는 Blitzkrieg, 방책 별로 성공 가능성 그래프를 지속적으로 최신화 해주고 시점별로 수행 내용을 구분해 주는 Cristal Ball이라는 3가지 하위체계로 구성되어 있다(Surdu \& Kittka, 2008).

우리 군의 경우, 인공지능을 활용하여 국방 의사결정 지원체계 구축방안에 대해 지휘통제체계를 중심으로 연구를 시작한 사례가 있으며, 인공지능 적용 소요 세부 내용을 Table 2 와 같이 판단하여 제시하고 있다. Table 2에서 소요되는 기능을 만들기 위해서는 사진과 영상 분석 및 처리에 적합한 딥러닝 기법이 적용되어야 표적을 식별하고 영상 분석이 자동적으로 이루어질 것으로 보이며, 방책 추천 및 모의 등을 위해서는 기존의 전사, 교리, 교범 등의 지식이 반영된 전문가 시스템을 이용해 야 할 것으로 보인다. 이런 체계를 대대부터 군단급의 전투지휘훈련 간에 활용하고 워게임 모델과 연계하여 발전시켜 나간다면, 우리 군의 지형, 교리, 전술에 맞는 의사결정 지원체계와 워게임 모델 의 구축이 가능할 것이다.

$<$ Table 2> Artificial Intelligence Applicants
A. multimedia reading
B. automatic analysis of target detection \& classification
C. enhancement of image analysis for understanding of situation in areas of operation
D. suggestion of course of action and simulation
E. intelligent searching support
F. report generation functions support
G. voice recognition functions support
H. translation functions support

* source: Korea Institute for Defense Analysis (2017)

\section{2 지능형 교관}

현재 학교 및 부대의 교육훈련은 1 명의 교관에 의해 다수의 인원을 가르치는 방식으로 진행되고 있다. 이런 전통적 교육방식보다 1:1의 교육이 효과적인 것은 자명한 사실이다. 그 이유는 개인별로 부족한 점을 파악하여 집중적으로 교육을 실시하는 개인별 맞춤 교육이 가능하기 때문이다. 하지만 개인별로 교관을 운용하는 것은 현실적으로 많은 제한사항이 있는데, 이를 보완할 수 있는 방안이 바로 지능형 교관(Intelligence Tutor) 시스템이다. 쉽게 말해 지능형 교관 시스템은 인간 교관의 개 입 없이 맞춤형 교육과 피드백을 학습자에게 제공해 주는 컴퓨터시스템이다.

우리 군의 모든 장병은 학습자이고, 군 생활 기간 동안 지속적으로 학습이 이루어진다. 지능형 교관은 학습자에게 지식을 전달하고, 학습자의 상태나 수준 등을 고려하여 학습 진도나 수준을 상 황에 맞추어 결정하여 최적의 학습여건을 보장할 수 있다. 학습자와 의사소통을 하는 지능형 교관 
의 모습은 웨어러블 기기나 로봇 또는 컴퓨터 등이 될 수 있을 것이다. 이런 시스템을 위해서는 군 사 분야 등의 도메인 지식, 학습자에 대한 정보 및 수준, 학습 전략 등 크게 3가지 요소에 대한 충분 한 자료가 필요하며, 이를 이용하여 학습자의 현재 지식구조를 진단하고 보완사항이 무엇인지 등 적절한 지도를 자동으로 해주는 알고리즘을 구현하는 것이 필요하다. 현재 사회에서도 이런 수준의 시스템 구축을 위해 교육정보시스템을 구축하기 위한 연구가 활발히 진행 중에 있다.

현재 야전에서 개인의 훈련 수준을 측정하고 결과를 유지하는 체계는 연대통합행정업무시스템 이 있으나, 정보 공유나 타체계 연동이 어렵고, 훈련 수준의 종합적인 판단이 제한되는 문제점이 있다. 더구나 부대의 훈련 수준을 측정하고 결과를 유지하는 체계는 거의 없는 실정이다. 한편, 학 교교육에서는 학습자의 수준 향상을 위해서 CBT(Computer Based Training)와 시뮬레이터 등을 활용하여 교육을 실시하고 있다. 하지만 현재 수준은 단편적인 지식과 장비조작 숙달 등이 대부분 이고, 획일적인 내용 등을 제공하기 때문에 개인별 수준에 맞는 교육은 이루어지기 어려운 상태이 다. 반면 지능형 교관 체계를 이용한다면, 야전 및 부대에서 개인별 수준을 고려하여 최적의 학습 효과를 달성할 수 있고, 개인과 부대의 훈련 수준이나 결과를 유지하면서 개선을 위한 피드백 제 공이 가능할 것이다. 즉, 학습자 중심의 교육훈련이 가능하므로 교육훈련 목표 달성이 용이해질 것이다.

이런 지능형 교관 시스템을 운용하기 위해 선행되어야 할 것은 학습자의 다양한 경험을 축적할 수 있는 개인별 교육정보시스템을 구축하기 위한 체계가 필요하다. 대표적인 데이터 수집 표준 체 계 중에 xAPI6)라는 것이 있는데, 이것에는 학습자의 성적기록, 진단내역, 학습기록, 피드백 정보 등의 자료를 구축하는 것이 가능하다. 군에서도 이와 유사한 시스템을 구축하여 병사 및 간부들의 정보를 구축하여 개인별로 부대 및 학교교육에 활용하는 교육이 필요하다.

\section{3 가상 개체 자동 모의}

스스로 상황을 인식하여 행동하는 가상의 개체가 필요한 부분은 시뮬레이터와 개체단위의 워게 임 모델이다. 시뮬레이터는 기능숙달, 전술훈련, 장비정비 등의 목적으로 사용된다. 전술훈련의 경 우 조종사가 인식하는 상황의 현실적인 묘사를 위해 대항군와 인접부대의 인원 및 전차 등의 개체 에 대한 자동 모의가 필요하다. 또한, 변화하는 전장상황을 고려하여 도시 내의 민간인 등에 대한 자동 모의도 필요할 것으로 보인다. 인원 및 전차 등의 개체 단위 자동 모의를 위해서는 현재 비디 오 게임에 활용되고 있는 인공지능 기법을 참고할 필요가 있다.

모델링 분야에서 실질적인 의사결정과 인간 행위를 자동으로 모의하는 것은 매우 중요하지만, 매우 어려운 일이다. 이를 위해 지금까지는 어떤 상태(state)와 전이(transition) 조건을 구성하고,

6) Experience Application Programing Interface의 약어로서 과거 학습의 기본 데이터뿐만 아니라 학습자의 다양한 경험에 바탕을 둔 프로그램을 의미함. 
Development and application of artificial intelligence (Al) for military training modeling and ... / Youngmook Kim 31

어떤 한 상태에서 조건이 충족되면 다른 상태로 이동하는 $\mathrm{FSM}^{7}$ )을 많이 사용하였다. 여기서는 $\mathrm{FSM}$ 보다 좀 더 발전되고 최근 군사용 게임이 많이 사용되고 있는 $\mathrm{HFSM}^{8}$ )과 BT9)에 대해 간략히 살펴보도록 한다(Evensen, Stien, \& Bentsen, 2018). 군사용 게임 중 하나인 VR-FORCE는 HFSM 을 사용하며, 마찬가지로 군사용 게임인 MASA-SWORD와 VBS3는 BT를 사용하고 있다. FSM은 특정 유형의 논리를 제공하는 것으로서 가장 단순한 예로 Figure 3의 좌측 그림처럼 상태는 이동, 교전, 후퇴의 3 가지가 있고, 조건이 충족되면 이동에서 교전으로, 교전에서 후퇴로 상태가 전이되는 개념이다. 이런 FSM은 상태가 많아지게 되면 매우 복잡해지고 수정이 어려운데, 이런 문제를 해 결하기 위해 Figure 3의 우측 그림과 같이 상태를 계층화하여 구현하는 개념인 $\mathrm{HFSM}$ 이 등장하게 되었다. HFSM은 확장성이 용이한 반면, 상태를 모듈화하고 재사용하는 것은 제한된다. 이런 제 한사항을 보완할 수 있는 개념이 BT라고 볼 수 있다. BT는 HFSM과 달리 전이가 구조에 의해 정의되기 때문에 노드들이 서로 독립적으로 설계할 수 있어 모듈화 및 확장성이 상대적으로 용 이하다.

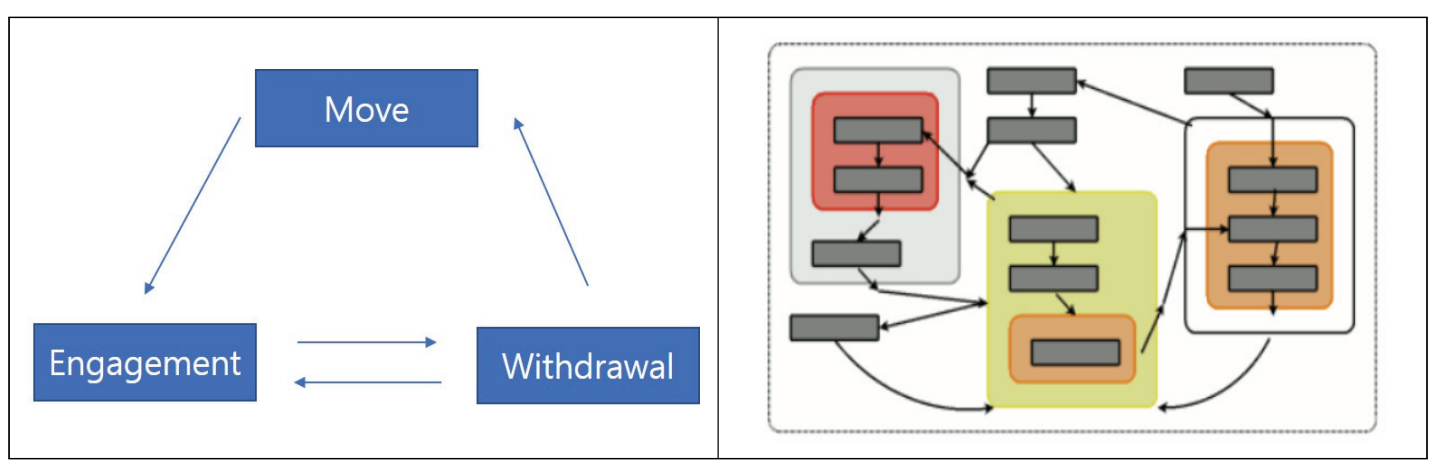

$<$ Figure 3> Concepts of FSM and HFSM

*source: from https://cps-vo.org/group/hfsm

최근 비디오 게임에서는 유지보수와 재사용성이 용이한 BT를 HFSM보다 많이 사용하고 있는 추세이다. 군에서 필요한 개체단위 자동모의를 위해서도 이런 게임 인공지능 기법 적용이 필요할 것으로 보이며, 이를 위해 북한군 및 우리 군의 교리와 민간인 행동이 반영된 상태 및 조건을 만드 는 노력이 필요하다. 또한, 훈련을 통해 얻은 자료를 분석하여 피드백한다면 보다 향상된 개체 단위 자동 모의를 구현하는 것이 가능할 것이다.

7) Finite State Machine(유한상태기계)

8) Hierarchical Finite State Machine(계층적 유한상태기계)

9) Behavior Tree(행동 트리) 


\section{V. 결론 및 논의}

지금까지 인공지능의 개념에 대해서 간단히 살펴보고, 이를 활용하고 있는 훈련 M\&S 분야 중 한국군의 창조21모델과 미군의 SAF에 대해서 알아보았다. 또한, 현재 대부분 워게임 모델 분야에 국한해서 적용하고 있는 인공지능 기법을 확대하여 적용할 수 있는 분야로서 의사결정 지원체계, 지능형 교관, 가상 개체 자동 모의 분야에 대해 살펴보았다. 훈련 M\&S 분야에서 이런 인공지능 기법들은 군사력 운용 측면에서 전투(전장) 환경을 고려한 시뮬레이션 교육훈련을 통해( $\mathrm{Bae}, \mathrm{Ku}$, Park, \& Kim, 2020) 편리하고 효과적인 도구가 될 수 있다. 따라서 앞으로 추가적인 연구가 필요한 부분은 각종 훈련별로 훈련의 목표와 대상을 명확히 설정한 뒤 기법들을 적용하는 데 필요한 방법 론 등을 구체화하는 것이다. 특수작전 부대원에 대해 도시지역 작전 훈련의 경우를 예로 살펴보자. 이런 훈련은 증강 또는 가상 현실을 이용하여 실시할 수 있어 훈련 환경 조성을 위해 도시 지역의 민간인을 모의해 주는 것이 필요하다. 이 때 자동 개체 자동 모의를 활용할 수 있으며, 부대원의 훈련 목표(민간인 밀집 상황에서 대테러 임무 수행 절차 숙달 등) 달성을 위해 민간인 가상 개체의 숫자와 다양한 상황 묘사가 가능한 민간인의 움직임 등에 관한 후속 연구가 필요하다.

2019년에 미국은 국방부에 JAIC(Joint Artificial Intelligence Center, 합동인공지능센터)를 설립 하였다. JAIC의 목표는 국방 전 분야에 걸쳐 인공지능을 적용하고, 기존에는 해결하기 어려웠던 문 제를 해결하며, 실시간으로 인공지능 관련 도구와 데이터의 접근이 가능한 체계를 구축하는 것이 다. 또한, JAIC에서는 최근 국방부 전 인원에 대해 인공지능 관련된 교육을 실시하여 국방 전 분야 에 인공지능의 적극적인 활용을 위한 노력을 실시하고 있다. 아직 우리나라에는 국방분야에는 $\mathrm{JAIC}$ 수준의 통합적인 인공지능 조직은 없는 실정이다.

앞에서 살펴본 국방분야에서 훈련 $\mathrm{M} \& \mathrm{~S}$ 분야의 인공지능 관련 발전방안은 하나의 군에만 필요 한 사항이 아니라 육, 해, 공군, 해병대 등 모든 군에 공통으로 필요한 사항이기 때문에 국방부 차원 에서 예산 투입과 기술개발 등이 필요하다. 이를 위해서는 미군의 JAIC 같은 조직인 국방부 수준에 서 필요하고, 이를 통해서 각 군의 체계와 자료 등을 활용할 수 있을 것이다.

한반도의 급변하는 안보 상황, 군복무 기간의 단축과 복무 인원 수 감소 등의 사회적 요인 등으 로 현 수준의 군사대비태세를 유지하기 위해서는 국방 분야에 인공지능 기법 적용은 반드시 필요 하다. 현재 인공지능 혁신의 중심에는 기계학습이 있다. 과거부터 존재한 기계학습이 현재 중심이 되는 이유 첫 번째는 우리가 접근할 수 있는 데이터가 급격히 증가했다는 것이고, 둘째는 이를 처 리할 수 있는 하드웨어가 발전했다는 것이다. 많은 데이터 처리에 적합한 딥러닝 기법 등이 큰 효 과를 보이는 것도 하나의 이유가 될 수 있다. 이런 기계학습의 특성상 훈련 M\&S 분야에 인공지능 기법을 활용하기 위해 필요한 것이 데이터 구축이다. 따라서 훈련 목표와 대상에 따른 방법론 구체 화와 더불어 관련된 데이터 구축도 병행되어야 한다. 국방부 차원에서 표준화되고 확장이 가능한 데이터 구축 시스템이 만들어지고, 현재의 발전된 인공지능 기법과 하드웨어를 활용하면 시간과 자 원을 절약하면서도 훈련 효과를 극대화하여 군사대비태세 확립에 크게 기여할 수 있을 것이다. 
Development and application of artificial intelligence (Al) for military training modeling and ... / Youngmook Kim 33

\section{Acknowledgements}

We would like to thank Editage (www.editage.co.kr) for English language editing.

\section{Declaration of Conflicting Interests}

The author(s) declared no potential conflicts of interest with respect to the research, authorship, and/or publication of this article. 


\section{Reference}

Ansari, Z., Azeem, M. F., Ahmed, W., \& Babu, A. V. (2015). Quantitative evaluation of performance and validity indices for clustering the web navigational sessions. arXiv preprint arXiv:1507.03340.

Arbin, N., Suhaimi, N. S., Mokhtar, N. Z., \& Othman, Z. (2015, December). Comparative analysis between $\mathrm{k}$-means and k-medoids for statistical clustering. In 2015 3rd International Conference on Artificial Intelligence, Modelling and Simulation (AIMS) (pp. 117-121). IEEE. https://doi.org/10.1109/AIMS.2015.82

Bae, S. H., Ku, X. L., Park, C. B., \& Kim, J. S. (2020). A Latent Topic Modeling approach for Subject Summarization of Research on the Military Art and Science in South Korea. Korean Journal of Military Art and Science, 76(2), 181-216. https://doi.org/10.31066/ kjmas.2020.76.2.008

Eom, H. S. (2021). A Study on the Combat Effectiveness of Dronebot Combat System using Lanchester's Law. Strategic Studies, 28(1),165-190. https://doi.org/10.46226/jss.2021.03. 28.1.165

Evensen, P. I., Stien, H., \& Bentsen, D. H. (2018). Modeling Battle Drills for Computer-Generated Forces using Behavior Trees. In Proceedings of the Interservice/Industry Training, Simulation and Education Conference (I/ITSEC) 2018. https://www.researchgate.net/ profile/Per-Idar-Evensen/publication/329416314_Modeling_Battle_Drills_for_Computer-Ge nerated_Forces_using_Behavior_Trees/links/5c079ae0a6fdcc315f9df158/Modeling-BattleDrills-for-Computer-Generated-Forces-using-Behavior-Trees.pdf

Han, S. K., Moon, H. S., Choi, Y. H., \& Yu, C. W. (2019). Study on the matching method of damage assessment caused by indirect fire between ChangJo21 and Combat21 models with different resolution. Journal of the Korean Data And Information Science Society, 30(5), 1105-1119. https://doi.org/10.7465/jkdi.2019.30.5.1105

Hill, R. R., \& Miller, J. O. (2017). A history of United States military simulation. 2017 Winter Simulation Conference (WSC). https:/doi.org/10.1109/wsc.2017.8247799

Kim, H. S., Moon, H. S., Lee, D. K., Hwang, M. S., \& Kim, Y. K. (2010). Developing an Expert System for Close Combat using Decision Tree. Journal of the Military Operations Research Society of Korea, 36(3), 83-93. UCI: G704-001703.2010.36.3.002

Korea Institute for Defense Analysis (2017, August). A Study on the Establishment of Defense Decision Support System Using AI, KIDA 3922, 21-8. https://www.kida.re.kr/frt/board/ 
Development and application of artificial intelligence (Al) for military training modeling and ... / Youngmook Kim 35

frtNormalBoard.do? wherecondition=itmval2\&itmval2=\&wherecondition=itmval3\&itmval3= \%EC\%A0\%95\%EB\%B3\%B4\%ED\%99\%94\&wherecondition=itmval4\&itmval4=\&pageIndex $=4 \&$ depth=2\&sidx $=701 \&$ stype $=$

Lee, Y., Park, M., \& Kim, Y. (2019). A Study on the Method for Converting the Unit Database from Training-model into Analysis-model : Focused on the 'Chang-Jo21' and 'Vision21' model. Journal of the Korea Society for Simulation, 28(2), 159-167. https://doi.org/ 10.9709/JKSS.2019.28.2.159

Miller, D. C., \& Thorpe, J. A. (1995). SIMNET: the advent of simulator networking. Proceedings of the IEEE, 83(8), 1114 - 1123. https://doi.org/doi:10.1109/5.400452

Moon, H. S. (2018). A study on an expert system for close combat battlefield situation assessment in war-game models using machine learning. Korean Journal of Military Art and Science, 74(3), 315-336. https://doi.org/10.31066/kjmas.2018.74.3.012

Surdu, J. R., \& Kittka, K. (2008, April). The deep green concept. In Proceedings of the 2008 Spring simulation multiconference (pp. 623-631). http://www.bucksurdu.com/Professional/ Documents/Case10307_TheDeepGreenConceptRevised.pdf

Sutton, R. S. (1992). Introduction: The Challenge of Reinforcement Learning. Reinforcement Learning, 1 - 3. https://doi.org/10.1007/978-1-4615-3618-5_1

Yoon, S., \& Choi, Y. (2020). Development of Defense Dronebot Integrated Control System: From battalion operations to Dronebot control and integrated control. Journal of Advances in Military Studies, 3(1), 35-50. https://doi.org/10.37944/jams.v3i1.65 
국문초록

https://doi.org/10.37944/jams.v4i2.114

\section{훈련 M\&S 분야 인공지능 적용 사례와 발전방안}

김영묵*

4차 산업혁명 시대를 맞이하여 인공지능을 활용하여 혁신을 이루고자 하는 노력이 사회 전반에 걸쳐 이루 어지고 있다. 국방분야에서도 이런 인공지능 기법을 이용하여 전투력 발휘를 극대화하기 위한 노력을 선진국 을 중심으로 실시하고 있다.

우리군에서 인공지능 관련 연구는 드론, 로봇 등 전장 관련된 부분에 집중되어 있으며, 상대적으로 훈련 분 야에 인공지능 기법 적용 연구는 부족한 실정이다. 이런 의미에서 본 연구는 인공지능의 개념에 대해 살펴보 고, 국방 M\&S 분야 중 하나인 훈련 M\&S 분야의 인공지능 기법을 활용하고 있는 사례를 분석하였다. 또한, 현재의 기술력을 이용하여 의사결정 지원체계, 지능형 교관, 가상개체 자동 모의에 대한 발전 방안을 제시하 였다.

주제어 : 인공지능, 훈련 M\&S, 의사결정 지원체계, 지능형 교관 시스템, 가상개체의 자동 모의

* (제1저자) 한미연합군사령부 소령(충남대학교 경영학과 박사과정), dudanr92@gmail.com 